LETTER TO JMG

\title{
The performance of $\mathrm{CGH}$ array for the detection of cryptic constitutional chromosome imbalances
}

\author{
J Schoumans, B-M Anderlid, E Blennow, B T Teh, M Nordenskjöld
}

J Med Genet 2004;41:198-202. doi: 10.1136/jmg.2003.013920

G ene dose alterations can cause mental retardation (MR), congenital malformations and miscarriages. Standard chromosome analysis by G-banding has a limited resolution, but molecular cytogenetic techniques, such as multi-subtelomeric FISH, microdeletion FISH, multicolour FISH and comparative genomic hybridisation (CGH), have played an important role for the diagnosis of MR during the past decade. ${ }^{1}$ A complete set of subtelomeric FISH probes was presented in 1996 and updated in $2000 .^{2}$ Consequently, screening for subtelomeric abnormalities has become a diagnostic test that is offered by diagnostic laboratories, and a number of studies reporting new subtelomeric rearrangements have been published. ${ }^{3-16}$ However, these probes only reveal chromosome rearrangements located in the subtelomeric region. To cover the whole genome, genome wide screening for chromosomal imbalances using microsatellite markers has been reported, ${ }^{17}{ }^{18}$ as well as metaphase CGH. ${ }^{19-22}$ Yet none of these techniques is able to offer a high resolution screening of the whole genome for chromosome imbalances. The development of accurate and sensitive genome wide screening methods would facilitate the clinical diagnosis of patients with very small or subtle rearrangements. Screening for chromosomal imbalances by array CGH, whether using $\mathrm{CDNA}^{23}$ or BAC clones, ${ }^{24}$ has mainly been performed on cancer samples, ${ }^{25-30}$ which usually contain large gene dose alterations. Although array CGH has provided a higher resolution compared to conventional CGH, it has not yet become a widely applied method for the analysis of gene dose alterations in individuals with idiopathic mental retardation. It has been a challenge to achieve the adequate performance needed for the reliable detection of single copy losses or gains of very small regions. Chromosome specific micro-arrays have however been used in a few cases to determine the critical regions in microdeletion disorders. ${ }^{31}{ }^{32}$ In this report we used a cDNA micro-array and two BAC clone micro-arrays on 10 previously identified cases containing 16 cryptic chromosome aberrations, to test their performance for the detection of small imbalances.

\section{MATERIALS AND METHODS}

Material selection

Ten cases with known cryptic aberrations were selected (seven cases with subtelomeric rearrangements, one case with an interstitial deletion and two cases with microdeletion syndromes: one case with DiGeorge syndrome and one case with a microdeletion of 17pll (Smith Magenis syndrome) in $60 \%$ of the lymphocytes). The 10 cases contained altogether 16 aberrations, with sizes ranging from 1.3 to $20.5 \mathrm{Mb}$, and they were located on 15 different chromosome arms. Routine chromosome analysis (450-500 bands) had failed to detect the rearrangements in all cases. Cases 2-8 (table 1) were identified using subtelomeric FISH probes. Cases 3-6 and 8 were previously published. ${ }^{63}$ Case 7 , with an interstitial deletion of 15q24, was serendipitously detected during subtelomeric screening, as the $15 \mathrm{q}$ control probe (LSI PML)

\section{Key points}

- Mental retardation is a common disorder, affecting 2$3 \%$ of the population, and independent lines of evidence point at chromosome imbalance as a major cause. The recent development of array based comparative genomic hybridisation (CGH) opens new opportunities for rapid high resolution genomic screening in genetic diagnostics.

- We have tested the robustness and simplicity of cDNA and bacterial artificial chromosome (BAC) microarrays on ten cases with previously identified cryptic chromosome aberrations, to determine if they might be introduced as reliable and sensitive diagnostic procedures.

- The test material contained 16 constitutional rearrangements, not detected by standard karyotyping, and all previously characterised by fluorescence in situ hybridisation (FISH) mapping. The aberrations ranged in size from 1.3 to $20.5 \mathrm{Mb}$.

- The cDNA array used contained 21632 cDNA clones and the BAC arrays contained 1000 and 2500 clones respectively.

- The cDNA array did not detect small heterozygous deletions or duplications.

- Using the BAC clone micro-array we detected all aberrations studied and were able to determine the size to $1 \mathrm{Mb}$ accuracy.

in the subtelomeric screening kit (Vysis Inc, Downers Grove, IL) was deleted. Cases 9 and 10 were detected using microdeletion FISH probes. Case 1 was identified by spectral karyotyping. Despite the large chromosome fragments involved in the unbalanced translocation in this case, repeated standard G-band analysis could not reveal this rearrangement due to the similarity in the banding pattern of the chromosome fragments involved. All chromosome imbalances were size mapped using BAC and PAC clones based on clone mapping databases (www.ensembl.org, July 2003), except for case 9, which was size mapped using a chromosome 22 specific micro-array. ${ }^{31}$

\section{FISH mapping}

Based on human clone mapping databases (www. ensembl.org, July 2003, www.genome.ucsc.edu, April 2003)

Abbreviations: BAC, bacterial artificial chromosome; $\mathrm{CGH}$, comparative genomic hybridisation; FISH, fluorescence in situ hybridisation; MR, mental retardation; PAC, P1 derived artificial chromosome 
206 BAC and Pl derived artificial chromosome (PAC) clones were selected for breakpoint mapping of the chromosome aberrations. The clones were obtained from Resources for Molecular Cytogenetics (Bari, Italy), The Wellcome Trust Sanger Institute (Cambridge, UK) and BACPAC Resource Center Children's Hospital, (Oakland Research Institute, Oakland, CA). Dual colour FISH was performed by hybridising each of the BAC clones to metaphase slides prepared from the selected patients as previously described. ${ }^{34}$

\section{DNA preparation, digestion, and labelling}

Genomic DNA was extracted from blood samples or EpsteinBarr virus transformed lymphocytes using Puregene blood kit (Gentra systems Inc, Minneapolis, MN) according to the manufacturer's protocol. Genomic DNA from the patients was digested into fragments of 100-2000 bp with DpnII (New England Biolabs Inc, Beverly, MA) and purified using Qiaquick PCR purification kit (Qiagen GmbH, Hilden, Germany). 0.8-2 $\mu \mathrm{g}$ of digested patient DNA, as well as reference DNA, was labelled by random priming with Cy5dCTP and Cy3-dCTP (Amersham Biosciences, Buckinghamshire, UK) respectively, using Bioprime labelling kit (Invitrogen, Carlsbad, CA).

\section{cDNA micro-array experiment}

cDNA micro-array production was performed within the Van Andel Institute Laboratory of Microarray Technology as described by Takahashi et al. ${ }^{35}$ Briefly, cDNA clones of the sequence verified human cDNA library (Research Genetics, Huntsville, AL) were PCR amplified directly from bacterial stock. The purified PCR products were robotically arrayed onto amino silane coated glass slides. Slides were blocked using succinic anhydride under standard conditions (http:// cmgm.stanford.edu/pbrown/). The arrays contained 21632 human cDNAs, generally with insert sizes of $0.25-2.5 \mathrm{~kb}$.

$0.8-2 \mu \mathrm{g}$ of labelled probes were mixed with $40 \mu \mathrm{g}$ human Cot-1 DNA (Invitrogen, Carlsbad, CA), before $16 \mu \mathrm{l}$ of $2 \times$ hybridisation solution $(50 \%$ formamide, $10 \times$ SSC, $0.2 \%$ SDS) was added. The probes were denatured at $100^{\circ} \mathrm{C}$ for $3 \mathrm{~min}$ and prehybridised at $37^{\circ} \mathrm{C}$ for $30 \mathrm{~min}$. After $16 \mathrm{~h}$ hybridisation at $42^{\circ} \mathrm{C}$ on the micro-array in a hybridisation chamber (Corning Inc, Corning, NY), the slides were washed in $1 \times \mathrm{SSC} / 0.1 \%$ SDS, $0.2 \times \mathrm{SSC} / 0.1 \%$ SDS and $0.1 \times \mathrm{SSC}$ for $5 \mathrm{~min}$ each at room temperature and immediately dried by centrifugation.

\section{BAC micro-array experiments}

We used two different commercially available genomic human BAC arrays (Spectral Genomics Inc, Houston, Texas). The arrays contained 1000 and 2500 BAC and PAC clones respectively, with all clones printed in duplicate. Hybridisation was performed according to the manufacture's protocol.

\section{Data analysis}

Arrays were scanned in a fluorescence confocal scanner (Scan Array Lite, GSI Lumonics, Billerica, CA). Images were analysed using GenePix Pro 3.0 software (Axon Instruments, Burlingame, CA). Spots were defined by the automatic grid feature of the software and adjusted manually when necessary. Further data handling was performed using Microsoft Excel. The fluorescence ratios across the array were normalised to achieve an average ratio of 1 for all cDNA or BAC elements on the array. Colour reverse experiments were performed for each patient sample to reduce variations related to labelling and hybridisation efficiencies. The mean Cy5/Cy3 ratios, standard deviation and co-efficient of variation of four replicas for each clone were calculated.
The cDNA micro-array consisted of 6292 known genes and 9944 unknown genes. For approximately 10000 of these 16236 different UniGenes, sequence accession numbers for each spot on the micro-array were mapped to UniGene cluster identifications and chromosome location using ftp:// ftp.ncbi.nlm.nih.gov/repository/UniGene (June 2002). Average ratios were calculated between fluorescent signals for each cDNA clone that clustered to the same gene and also from the colour reverse experiments.

The BAC clone arrays were analysed by calculating the average ratio between four fluorescent signals (two signals from the duplicated clone on the array and two signals from the colour reverse experiment) and the fluorescence ratios were plotted according to their mapped location (http:// www.ncbi.nlm.nih.gov/mapview/ (March 2003)). Clones displaying a standard deviation $>0.20$ were interpreted as hybridisation failures and were excluded from the BAC array analysis. DNA copy number profiles $>1.2$ were scored as gained, $<0.80$ as lost, as previously described. ${ }^{1025}$

\section{RESULTS}

The data for each case are summarised in table 1. Using the cDNA array we detected $50 \%$ of the aberrations ( 7 out of 14). The smallest detected aberration by this method was a terminal deletion of $8.7 \mathrm{Mb}$ on chromosome $4 \mathrm{q}$ (fig lA). The results from the cDNA arrays were difficult to interpret because of numerous false positive and false negative findings. The aberrations were only detectable when the $\mathrm{Cy} 5 / \mathrm{Cy} 3$ ratio values from a large number of clones located next to each other on the physical map deviated from ratio 1 (normal). Fig lA illustrates the detection of the unbalanced rearrangements of case 5 with a duplication of $2 \mathrm{q}$ and a deletion of $4 \mathrm{q}$, barely detectable by the cDNA array.

The results of the BAC clone micro-array containing 1000 clones were confusing (data not shown). False positive and false negative results were detected using this array and the smallest aberration detected was a $3.5 \mathrm{Mb}$ deletion on chromosome 6q (case 6), while a large duplication on chromosome 9q (case 3) was not detected. This can be explained by errors in the physical mapping of the clones, which resulted in chromosome regions that were not well covered (9qter had a gap of $5.3 \mathrm{Mb}$ ). In addition, one clone on 9qter showed significant homology to chromosome 13 and three clones on 9qter gave false negative results due to weak specific signals.

The 2500 clone array detected all of the aberrations studied and their sizes could also be determined by approximately $1 \mathrm{Mb}$ accuracy (table 1). No false positive results were observed. However, false negative findings were observed. Nine clones located in the subtelomeric regions of the aberrant chromosomes, four clones on 10q (AL392043.1, RP11-90B19, GS-137-E24, GS-261-B16), two clones on 17q (AQ285007, GS-362-K4), one clone on 12q (RP11-119J21), one clone on 6p (AL035696.14), and one clone on 9q (RP11$89 \mathrm{P} 10$ ) showed a ratio close to 1 (normal). In addition, two clones on the X chromosome (CTB-188I17, AL031643.1) did not show the expected ratio gain $(>1.2)$ when a female sample was hybridised against a male reference, or loss $(<0.8)$ when a male sample was hybridised against a female reference. Clones AL031643.1 and CTB-188117 were tested for cross-hybridisation by FISH. Clone AL031643.1 hybridised weakly but specifically to chromosome Xp21.1. However, CTB-188I17 was not X-specific, since it also hybridised on the Y chromosome (data not shown). All aberrations from cases 1,4 , and 6 to 10 were also correctly detected in a blind analysis performed by Spectral Genomics using a 1400 BAC array (data not shown). 


\begin{tabular}{|c|c|c|c|c|c|c|}
\hline Case & $\begin{array}{l}\text { Chromosome } \\
\text { Abnormality }\end{array}$ & $\begin{array}{l}\text { CDNA } \\
\text { array }\end{array}$ & $\begin{array}{l}2500 \text { clone } \\
\text { array }\end{array}$ & $\begin{array}{l}\text { size in Mb by } \\
\text { FISH mapping }\end{array}$ & $\begin{array}{l}\text { size estimation by } \\
1 \mathrm{Mb} \text { array in } \mathrm{Mb}\end{array}$ & $\begin{array}{l}\text { number of del/dup } \\
\text { clones on } 1 \mathrm{Mb} \text { array }\end{array}$ \\
\hline \multirow[t]{2}{*}{1} & monosomy $7 q$ & D & D & 13.2 & 14 & 9 \\
\hline & trisomy $10 q$ & D & D & 19.1 & 20 & 22 \\
\hline \multirow[t]{2}{*}{2} & monosomy $18 p$ & ND & D & 5.8 & 6 & 4 \\
\hline & trisomy $13 q$ & ND & D & 5.9 & 6 & 6 \\
\hline \multirow[t]{2}{*}{3} & monosomy $21 \mathrm{q}$ & ND & D & 2.5 & 3 & 2 \\
\hline & trisomy $9 q$ & D & D & 11.2 & 12 & 13 \\
\hline \multirow[t]{2}{*}{4} & monosomy $12 q$ & ND & D & 2.1 & 2 & 1 \\
\hline & trisomy $17 q$ & ND & D & 1.3 & 1 & 1 \\
\hline \multirow[t]{2}{*}{5} & monosomy $4 \mathrm{q}$ & D & D & 8.7 & 9 & 10 \\
\hline & trisomy $2 q$ & D & D & 20.5 & 20 & 22 \\
\hline \multirow[t]{2}{*}{6} & monosomy 69 & ND & D & 3.5 & 4 & 6 \\
\hline & trisomy op & $D$ & D & 14.7 & 16 & 21 \\
\hline 7 & deletion $15 \mathrm{q} 24$ & D & D & 10.3 & 10 & 8 \\
\hline 8 & deletion $6 p$ & ND & D & 1.8 & 1.8 & 5 \\
\hline 9 & deletion $22 \mathrm{q} 11.2$ & - & D & 2.5 & 3 & 3 \\
\hline 10 & deletion $17 p 11$ & - & D & $3.6^{*}$ & $\sim 4$ & 2 \\
\hline
\end{tabular}

\section{DISCUSSION}

Reliable and high-resolution detection of copy number changes of genomic DNA is fundamental in diagnosing patients with mental retardation and chromosome imbalances in cancer. CGH makes it possible to screen the whole genome for genomic imbalances. However, CGH performed on metaphase chromosomes has a comparatively low resolution (5-10 Mb) and the resolution is likely to depend on the chromosome region involved. The use of cDNA microarrays was not very successful in delineating chromosome changes and there may be several explanations for these results. Firstly, cDNA micro-arrays were primaryily developed for expression profiling. The cDNA sequences are therefore mostly selected based on gene function, not on chromosome location, which results in poor coverage of some chromosome regions. Secondly, we found it difficult to interpret the
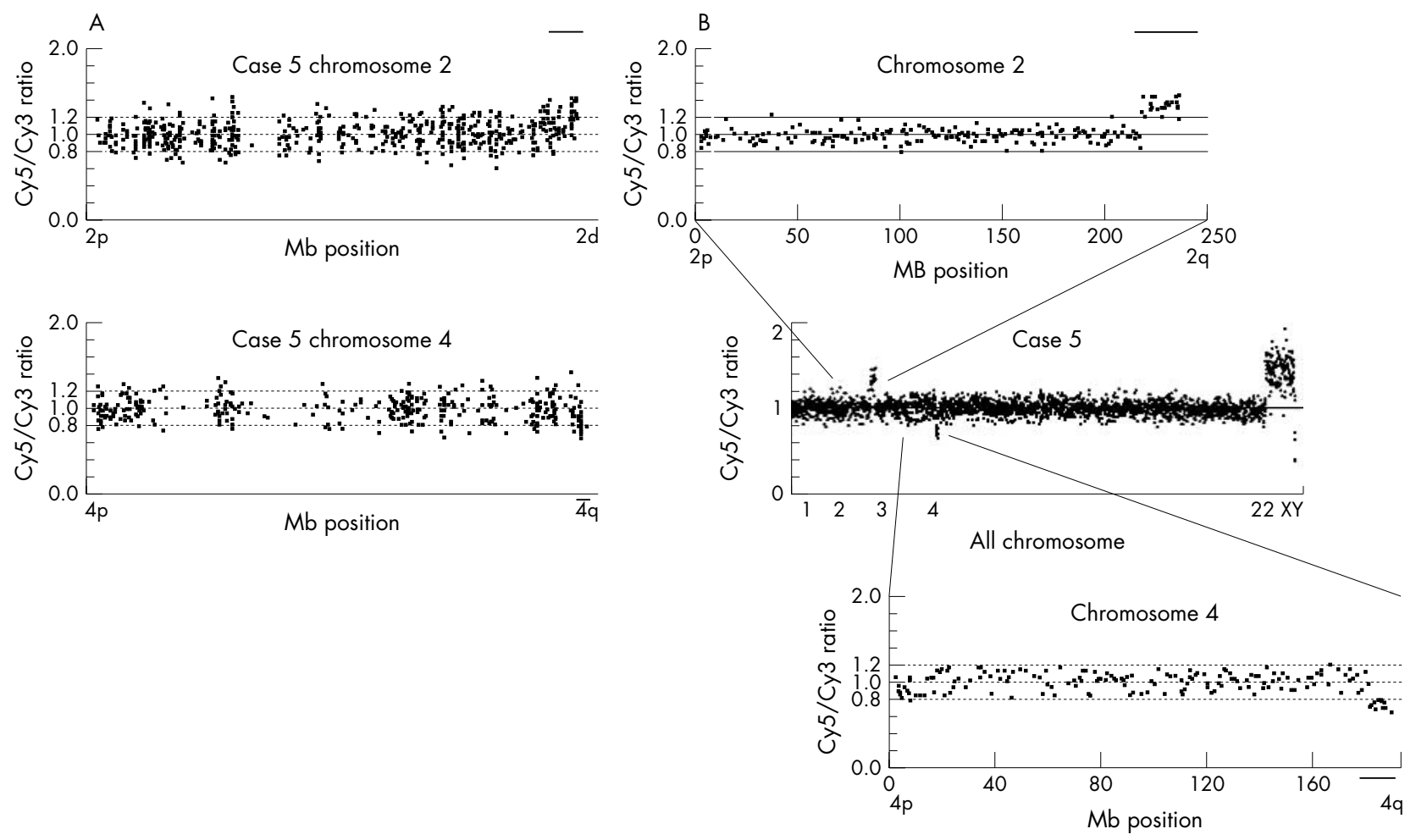

Mb position

Figure 1 A) Ratio plot from Human cDNA micro-array data from chromosome 2 and 4 (case 5). Each dot on the chart represents the normalised, average ratio between fluorescent signals from colour reverse experiment for each cDNA sequence that cluster to the same gene. The dots are linearly ordered along the chromosome according to their $\mathrm{Mb}$ location, starting with pter to the left and qter to the right. The plot shows a duplication of 2 gter, and a deletion of 4 qter is barely visible. Not all chromosome regions are well represented, especially on chromosome 4, and many ratios deviate from a modal value of 1.0, which complicates the interpretation. B) Ratio plot from Human BAC micro-array data, containing 2500 clones from all chromosomes with a magnification of ratio plot for chromosome 2 and 4 (case 5). The dots are linearly ordered along all chromosomes according to their Mb location, starting with chromosome 1 to the left and the Y chromosome to the right. The plot shows a clear duplication of 2qter, a clear deletion of 4 qter, a duplication of the $X$ chromosome and a deletion of $Y$ (the patient DNA is hybridised against reference DNA of the opposite sex). Spots showing ratios $\geqslant 0.8$ and $\leqslant 1.2$ are considered to be normal. 
hybridisation results, because of poor specific signal to noise ratios. The higher background observed with the cDNA arrays, as compared with BAC arrays, was probably due to the differences in length of the DNA sequences spotted on the array. ${ }^{24}$ The use of large BAC clone sequences results in a more specific signal. Other reports also conclude that single ESTs are not reliable targets in array CGH experiments for the detection of single DNA copy changes. ${ }^{36}$ Thirdly, the slides were coated to attach the cDNA on the glass surface, a strategy that introduces hybridisation background noise.

Several different strategies to improve the specific signal to noise ratio have been reported during the past year-for example the repeat free and non-redundant strategy ${ }^{31}$ and the use of specifically designed DOP-PCR primers for amplification of the BAC clones. ${ }^{26}$ Regarding the BAC micro-arrays used in this study, the DNA of the BAC clones was not PCR amplified but purified in large scale from culture and the nucleic acids were attached onto an uncoated glass surface by using chemical coupling. ${ }^{25}$ This increases the sensitivity and lowers the background signal.

In conclusion, the human BAC clone micro-array works very well for the detection of cryptic chromosome rearrangements. In our hands the method was very robust. It is also very useful for size mapping of the aberrations, which facilitates the phenotype-genotype correlation. It is therefore likely that the CGH array will be offered as a genetic test in clinical diagnostic laboratories in the near future. Regular CGH and CDNA arrays are limited in sensitivity and robustness and are therefore not as reliable as the BAC array. False positive and false negative results have also been a large concern when using BAC arrays for genomic screening for single copy detection. We experienced these problems using the 1000 clone BAC array, but the improved 2500 clone arrays did not show any false positive results, and only 11 clones showed false negative results. There are several plausible explanations for the fact that these clones showed fluorescence ratios deviating from the expected values for one copy loss or gain. Segments of these clone sequences can be highly similar to sequences present elsewhere in the genome (for example, clone CTB-188I7 showed not to be X-specific) and some clones might contain large segments of commonly shared repeat sequences. These sequences are suppressed by Cot-1 DNA during prehybridisation, but it reduces the specific signal intensity while incomplete suppression can result in non-specific signals. Finally, some of the clones might still be incorrectly assigned on the physical map.

In our study, we did not experience any problems in the detection or interpretation of the size of the aberrations studied, using the 2500 clone array despite the fact that 11 clones failed to detect the duplication or deletion, since they all had a size of at least $1 \mathrm{Mb}$. For the detection of small chromosome aberrations $(\leqslant 1 \mathrm{Mb})$, the array needs further improvement, by replacing the unstable clones and by adding more clones.

In the very near future, all elements of BAC libraries will be completely characterised and mapped, a fact that will further improve the reliability of BAC micro-arrays. 32000 selected human BAC clones containing the draft sequence of the human genome can be ordered (http://bacpac.chori.org/ pHumanMinSet.htm) to manufacture a micro-array that fully covers the genome with a theoretical resolution that is only limited by the size of the BACs. When BAC arrays are introduced in diagnostic laboratories, genetic changes that were previously unknown will be detected and new genomic imbalances and syndromes will be described. In addition, normal variants will probably also be found, as was the case when subtelomeric screening was widely performed. ${ }^{11}{ }^{13}$

\section{ACKNOWLEDGEMENTS}

We thank Xiang Guo for his statistical expertise, Isabel Tapia Páez for the chromosome 22 specific array data and the Laboratory of DNA and Protein Microarray technology, Van Andel Research Institute, for providing the cDNA arrays. We also want to thank Resources for Molecular Cytogenetics, Bari, the Wellcome Trust Sanger Institute and BACPAC Resource Center, Children's Hospital Oakland Research Institute for providing $\mathrm{BAC}$ and PAC clones.

\section{Authors' affiliations}

J Schoumans, B-M Anderlid, E Blennow, M Nordenskjöld, Department of Molecular Medicine, Clinical Genetics Unit, Karolinska Institute, CMM L8:02 Karolinska Hospital, SE-17176 Stockholm, Sweden

B T Teh, Laboratory of Cancer Genetics, Van Andel Research Institute, Grand Rapids, Ml 49503, USA

This work was supported by funds from the Swedish Medical Research Council, Frimurarna Barnahuset Foundation, and the Ronald McDonald Child Foundation.

Correspondence to: Jacqueline Schoumans;

Jacqueline.schoumans@cmm.ki.se

Received 3 September 2003

In revised form 16 September 2003

Accepted 25 September 2003

\section{REFERENCES}

1 Xu J, Chen Z. Advances in molecular cytogenetics for the evaluation of mental retardation. Am J Med Genet 2003;117C:15-24.

2 Knight SJ, Flint J. Perfect endings: a review of subtelomeric probes and their use in clinical diagnosis. J Med Genet 2000;37:401-9.

3 Clarkson B, Pavenski K, Dupuis L, et al. Detecting rearrangements in children using subtelomeric FISH and SKY. Am J Med Genet 2002;107:267-74.

4 Baker E, Hinton L, Callen DF, et al. Study of 250 children with idiopathic mental retardation reveals nine cryptic and diverse subtelomeric chromosome anomalies. Am J Med Genet 2002;107:285-93.

5 Fan YS, Zhang Y, Speevak M, et al. Detection of submicroscopic aberrations in patients with unexplained mental retardation by fluorescence in situ hybridization using multiple subtelomeric probes. Genet Med 2001;3:416-21.

6 Anderlid BM, Schoumans J, Anneren G, et al. Subtelomeric rearrangements detected in patients with idiopathic mental retardation. Am J Med Genet 2002; 107:275-84.

7 Knight SJ, Regan R, Nicod A, et al. Subtle chromosomal rearrangements in children with unexplained mental retardation. Lancet 1999;354:1676-81.

8 Sismani C, Armour JA, Flint J, et al. Screening for subtelomeric chromosome abnormalities in children with idiopathic mental retardation using multiprobe telomeric FISH and the new MAPH telomeric assay. Eur J Hum Genet $2001 ; 9: 527-32$

9 Slavotinek A, Rosenberg M, Knight S, et al. Screening for submicroscopic chromosome rearrangements in children with idiopathic mental retardation using microsatellite markers for the chromosome telomeres. J Med Genet 1999;36:405-11.

10 Veltman JA, Schoenmakers EF, Eussen BH, et al. High-throughput analysis of subtelomeric chromosome rearrangements by use of array-based comparative genomic hybridization. Am J Hum Genet 2002;70:1269-76.

11 Van Karnebeek CD, Koevoets C, Sluijter S, et al. Prospective screening for subtelomeric rearrangements in children with mental retardation of unknown aetiology: the Amsterdam experience. J Med Genet 2002;39:546-53.

12 Hollox EJ, Atia T, Cross G, et al. High throughput screening of human subtelomeric DNA for copy number changes using multiplex amplifiable probe hybridisation (MAPH). J Med Genet 2002;39:790-5.

13 Joyce CA, Dennis NR, Cooper S, et al. Subtelomeric rearrangements: results from a study of selected and unselected probands with idiopathic mental retardation and control individuals by using high-resolution G-banding and FISH. Hum Genet 2001; 109:440-51.

14 Brown J, Saracoglu K, Uhrig S, et al. Subtelomeric chromosome rearrangements are detected using an innovative 12-color FISH assay (MTEL). Nat Med 2001;7:497-501.

15 Dawson AJ, Putnam S, Schultz J, et al. Cryptic chromosome rearrangements detected by subtelomere assay in patients with mental retardation and dysmorphic features. Clin Genet 2002;62:488-94.

16 Popp S, Schulze B, Granzow M, et al. Study of 30 patients with unexplained developmental delay and dysmorphic features or congenital abnormalities using conventional cytogenetics and multiplex FISH telomere (M-TEL) integrity assay. Hum Genet 2002;111:31-9.

17 Rosenberg MJ, Vaske D, Killoran CE, et al. Detection of chromosomal aberrations by a whole-genome microsatellite screen. Am J Hum Genet 2000;66:419-27.

18 Rio M, Molinari F, Heuertz S, et al. Automated fluorescent genotyping detects $10 \%$ of cryptic subtelomeric rearrangements in idiopathic syndromic mental retardation. J Med Genet 2002;39:266-70. 
19 Kirchhoff $M$, Rose $\mathrm{H}$, Lundsteen $\mathrm{C}$. High resolution comparative genomic hybridisation in clinical cytogenetics. J Med Genet 2001;38:740-4.

20 Joly G, Lapierre JM, Ozilou C, et al. Comparative genomic hybridisation in mentally retarded patients with dysmorphic features and a normal karyotype. Clin Genet 2001:60:212-9.

21 Sanlaville D, Romana SP, Lapierre JM, et al. A CGH study of 27 patients with CHARGE association. Clin Genet 2002;61:135-8.

22 Ness GO, Lybaek H, Houge G. Usefulness of high-resolution comparative genomic hybridization (CGH) for detecting and characterizing constitutional chromosome abnormalities. Am J Med Genet 2002;113:125-36.

23 Pollack JR, Perou CM, Alizadeh AA, et al. Genome-wide analysis of DNA copy-number changes using cDNA microarrays. Nat Genet 1999;23:41-6.

24 Snijders AM, Nowak N, Segraves R, et al. Assembly of microarrays for genomewide measurement of DNA copy number. Nat Genet 2001;29:263-4.

25 Cai WW, Mao JH, Chow CW, et al. Genome-wide detection of chromosomal imbalances in tumors using BAC microarrays. Nat Biotechnol 2002;20:393-6.

26 Fiegler $\mathrm{H}$, Carr $\mathrm{P}$, Douglas EJ, et al. DNA microarrays for comparative genomic hybridization based on DOP-PCR amplification of BAC and PAC clones. Genes Chromosomes Cancer 2003;36:361-74.

27 Heiskanen MA, Bittner ML, Chen Y, et al. Detection of gene amplification by genomic hybridization to cDNA microarrays. Cancer Res 2000:60:799-802.

28 Hodgson G, Hager JH, Volik S, et al. Genome scanning with array CGH delineates regional alterations in mouse islet carcinomas. Nat Genet $2001 ; 29: 459-64$
29 Lucito R, West J, Reiner A, et al. Detecting gene copy number fluctuations in tumor cells by microarray analysis of genomic representations. Genome Res 2000;10:1726-36

30 Veltman JA, Fridlyand J, Pejavar S, et al. Array-based comparative genomic hybridization for genome-wide screening of DNA copy number in bladder tumors. Cancer Res 2003;63:2872-80.

31 Buckley PG, Mantripragada KK, Benetkiewicz M, et al. A full-coverage, highresolution human chromosome 22 genomic microarray for clinical and research applications. Hum Mol Genet 2002;11:3221-9.

32 Veltman JA, Jonkers $Y$, Nuiiten I, et al. Definition of a critical region on chromosome 18 for congenital aural atresia by array-CGH. Am J Hum Genet 2003;72:1578-84.

33 Anderlid BM, Schoumans J, Hallqvist A, et al. Cryptic subtelomeric 6p deletion in a girl with congenital malformations and severe language impairment. Eur J Hum Genet 2003;11:89-92.

34 Pinkel D, Gray JW, Trask B, van den Engh G, Fuscoe J, van Dekken H. Cytogenetic analysis by in situ hybridization with fluorescently labeled nucleic acid probes. Cold Spring Harb Symp Quant Biol 1986:51:151-157.

35 Takahashi $M$, Rhodes DR, Furge KA, et al. Gene expression profiling of clear cell renal cell carcinoma: gene identification and prognostic classification. Proc Natl Acad Sci U S A 2001;98:9754-9.

36 Beheshti B, Braude I, Marrano P, et al. Chromosomal localization of DNA amplifications in neuroblastoma tumors using CDNA microarray comparative genomic hybridization. Neoplasia 2003;5:53-62.

\section{$\mathrm{ECHO}$}

\section{Standardised measures of retinal function pinpoint genotype in X linked congenital stationary night blindness}

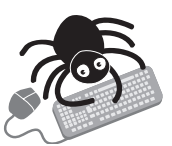

Please visit the Journal of Medical Genetics website [www. imedgenet. com] for a link to the full text of this article.
- ye specialists using standardised electrophysiological criteria for the first time have

E suggested that these are more reliable indicators of genotype in X linked congenital - stationary night blindness (CSNBX) than those used formerly.

Just three measures separated 20 affected males from 11 British families with CSNBX, according to whether they had mutations in NYX or CACNAIF genes. NYX mutations resulted in absent scotopic oscillating potentials, CACNAIF mutations in subnormal OFF response, and each mutation had different wave forms and amplitudes in $30 \mathrm{~Hz}$ flicker tests. Other indicators of eye function were not specific enough.

Three of the families had CACNAIF mutations, each with a different mutation, one of which was a novel nonsense mutation in exon 7 . The others were a nonsense mutation in exon 24 and a base pair (bp) deletion in exon 9. All were expected to produce a truncated protein product. The eight remaining families had five NYX mutations: a splicing mutation; a missense and a nonsense mutation predicting truncated protein product; a $15 \mathrm{bp}$ in frame deletion; and a 335 bp deletion.

Affected males were prospectively clinically evaluated for visual disorders and by psychophysiological and electrophysiological testing in parallel with genotyping.

Until now, non-standardised testing has subdivided CSNBX phenotype into "complete" and "incomplete" forms, apparently associated with NYX and CACNAIF mutations, respectively. However this distinction has proved unsatisfactory, in the light of reports of a functional rod pathway in patients with NYX mutations and of complete and incomplete forms of the condition in the same family.

A British Journal of Ophthalmology 2003;87:1413-1420. 